\title{
Longitudinal genome-wide analysis of patients with chronic lymphocytic leukemia reveals complex evolution of clonal architecture at disease progression and at the time of relapse
}

\author{
E Braggio ${ }^{1}$, NE Kay ${ }^{2}$, S VanWier ${ }^{1}$, RC Tschumper ${ }^{3}$, S Smoley ${ }^{4}$, JE Eckel-Passow ${ }^{5}$, T \\ Sassoon ${ }^{2}$, M Barrett $^{6}$, DL Van Dyke ${ }^{4}$, JC Byrd ${ }^{7}$, DF Jelinek ${ }^{3}$, TD Shanafelt ${ }^{2}$, and R Fonseca ${ }^{8}$ \\ R Fonseca: fonseca.rafael@mayo.edu \\ ${ }^{1}$ Department of Biochemistry and Molecular Biology, Mayo Clinic, Scottsdale, AZ, USA \\ ${ }^{2}$ Division of Hematology and Internal Medicine, Mayo Clinic, Rochester, MN, USA \\ ${ }^{3}$ Department of Immunology, Mayo Clinic, Rochester, MN, USA \\ ${ }^{4}$ Department of Laboratory Medicine and Pathology, Division of Laboratory Genetics, Mayo Clinic, \\ Rochester, MN, USA \\ ${ }^{5}$ Division of Biomedical Statistics and Informatics, Mayo Clinic, Rochester, MN, USA \\ ${ }^{6}$ Translational Genomics, Phoenix, AZ, USA \\ ${ }^{7}$ Department of Hematology, Ohio State University, Columbus, OH, USA \\ ${ }^{8}$ Department of Hematology - Oncology, Mayo Clinic, Scottsdale, AZ, USA
}

\begin{abstract}
Recent advances in comprehensive genomic analyses, which include array-based comparative genomic hybridization (aCGH) and massively parallel sequencing, have permitted a better appreciation of the genetic complexity and clonal heterogeneity of the human tumor cell population. ${ }^{1-8}$ Although such analyses of tumor cells have provided evidence of extraordinary genomic complexity and intra-clonal genetic diversity in several cancers, little is known of the genetic architecture and intra-clonal heterogeneity of the leukemic clone in chronic lymphocytic leukemia (CLL) patients, ${ }^{9-11}$ and no sequential global genomic analyses have been performed to better understand the process of clonal evolution associated with the tumor cells in this malignancy. In order to gain insight into the genetic complexity and clonal evolution underlying disease progression/relapse, we performed a sequential, longitudinal analysis of tumor samples in 22 CLL patients.
\end{abstract}

All 22 patients had genome-wide genetic analysis performed by aCGH on at least two sequential tumor samples $>6$ months apart (54 samples from 22 patients with two to four time points (TPs) analyzed per patient). For the purposes of this study, we classified samples as; TP1 collected > 6 months before starting first-line treatment: TP2 consisting of tumor samples at the time of progression requiring treatment and TP 3 consisting of tumor samples

(C) 2012 Macmillan Publishers Limited

CONFLICT OF INTEREST

$\mathrm{RF}$ is a consultant for Genzyme, Medtronic, BMS, Amgen, Otsuka, Celgene, Intellikine and Lilly (all less than \$10 000). RF receives research support from Cylene, Onyx and Celgene. The remaining authors declare no conflict of interest.

AUTHOR CONTRIBUTIONS

EB, NK, TDS, RF and DFJ designed the study; EB, SVW, SS, JEP, DVD MB, TS and RCT performed the research; EB wrote the paper and all authors reviewed and gave final approval of the manuscript.

Supplementary Information accompanies the paper on the Leukemia website (http://www.nature.com/leu) 
collected $>6$ months after initial treatment but before subsequent treatments. ${ }^{12}$ In cases showing genetic changes between TPs, fluorescence in situ hybridization (FISH) analyses were performed. Demographics, TPs analyzed and the technical approach used in each case are summarized in Supplementary Material, Supplementary Table S2 and Figure S1.

Overall, 6 of 22 patients (27\%) showed copy-number abnormalities (CNA) differences between TPs. There was a small increase in genomic complexity in later TPs with a mean of 4 CNAs (median 4, range 0-12) detected at the first TP, increasing to a mean of 5.3 CNAs (median 4.5, range 0-21) at the last TP analyzed (Supplementary Table S3). Trisomy 12 and deletion 11q32 were stable over time, whereas there was an incremental in the number of cases with deletion $13 \mathrm{q} 14.3$ and $17 \mathrm{p}$ in more advanced stages of the disease.

In the six cases with CNA changes between TPs, FISH analyses were performed for validation and to estimate the percentage of B cells with the different CNAs. Diverse patterns of changes in the genetic architecture were observed. The simplest change was the linear sequence, characterized by the maintenance of the initial abnormalities and the subsequent acquisition of additional CNAs in the same subclone (Figure 1a). This linear evolution of clone architecture was found in two cases (patients 9 and 17). Patient 9 had five CNAs at TP1 and acquired 9 additional CNAs (including deletion 13q14.3) at TP2. These nine CNAs were not found at TP1 using follow up FISH analysis, thus confirming that they were either absent or present in $<5 \%$ of cells (that is, FISH detection threshold) at early stages of the disease. Patient 17 showed 12 CNAs at TP1 and acquired 9 additional CNAs at TP2. Additionally, a TP53 frame shift deletion (D148> $\times 168$ ) was found at TP2 (Figure 1a). None of these nine CNAs or the TP53 mutation were found at TP1.

The pattern of changes in the genetic architecture for the remaining four cases demonstrated marked heterogeneity with two or more subclones identified. Patient 8 was characterized by the presence of three subclones. All subclones shared trisomy 12 and gain of 19p13.2q13.12, but also displayed several subclone-specific CNAs (Figures 1b-d). The subclone 'A' has seven additional CNAs, including deletions of 13q13.1 (RBI) and 13q14.3 (MIRN15A and MIRN16-1). Six different CNAs were specific to the subclone 'B', including heterozygous deletion of 13q14 (MIRN15A/MIRN16-1). Subclone ' $\mathrm{C}$ ' likely was originated from subclone 'B' by acquiring homozygous deletion of 13q14.3 (Figure 1d). Subclone 'A' was the dominant clone at TP1 and TP2, found in $~ 70 \%$ of tumor cells. At TP3, subclone 'A' was detected in only $20 \%$ of the tumor cells. Conversely, subclone 'B', which was present in $\sim 25-30 \%$ of tumor cells at TP1 and TP2, became the dominant clone at TP3, observed in $\sim 60 \%$ of the leukemic cells. Subclone ' $\mathrm{C}$ ' also increased in prevalence over time from $5 \%$ in TP1 to $20 \%$ in TP3 (Figure 1d).

A similar complex change in the genetic architecture was found in patient 22, with two genetically defined subclones sharing the trisomy 12 but also showing subclone-specific abnormalities. Subclone 'A' was observed in 70\% of cells at TP2, whereas subclone 'B' was observed in $30 \%$ of cells. At TP3, however, subclone 'B' became the dominant subclone present in $\sim 90 \%$ of leukemic cells.

In the remaining two patients with changes in the genetic architecture, no apparent common CNAs were found between (sub)clones. Patient 16 showed a sole deletion on 11q22.3-q23.2 at TP2. The karyotype became more complex at TP3, with deletion of 11q22.3-q23.2 and six additional CNAs, including gains of $2 \mathrm{p} 16$ and 9q34, and deletions of 9p21, 9q12-q33, $14 q 13.2$ and 17 p (Figure 1e). Furthermore, a missense mutation was found in the remaining TP53 allele (D281N) at TP3. Interestingly, the deletions 11q22.3-q23.2 observed at both TPs were not related in origin as was confirmed by the presence of different chromosomal breakpoints (Figure 1f). A FISH assay designed to cover the differentially deleted area 
confirmed the aCGH finding that two independent abnormalities affected 11q22.3-q23.2 in the separate TPs. In addition, a different copy-number variant pattern was found in the deleted region between samples, confirming that separate and different chromosome 11 sources (maternal versus paternal) were affected in the two subclones (Figure 1f). This finding illustrates the importance of genome-wide analysis, as FISH alone would not have identified the existence of two unrelated clones in this case.

Finally, at least three subclones were identified on patient 13. Two of these subclones exhibited a linear related sequence (clone ' $\mathrm{B}$ ' and its derivative clone ' $\mathrm{C}$ '), however, the remaining (clone ' $\mathrm{A}$ ') had no apparent genetic relationship with these two subclones.

In all four patients with multiple subclones, a marked shift in the dominant subclone was observed at the time of disease relapse, with the minor subclones at TP1 and TP2 becoming the dominant subclones at TP3 (Figure 2). In addition, the subclonal evolution continued following secondary therapies, and in all four cases the emerging subclone remains dominant after multiple treatments. This argues in favor of subclones with specific genetic structures becoming dominant with the selective evolutionary pressure imposed by therapy.

To our knowledge, the extent to which cancer cells differentiate genetically at the subclonal level has not been comprehensively tested in CLL. This study refines our understanding of the dynamic genetic changes in progressive CLL and offers a better understanding of the clonal evolution that can occur in an individual patient before and after therapy. Our analysis reveals a previously unappreciated genetic heterogeneity present in CLL, where many patients exhibited a variable number of genetically distinct subclones. Collectively, $>25 \%$ of CLL patients exhibited changes in the genetic architecture and at least $18 \%$ showed evidence of multiple subclones, although we suspect that the mutation screening by whole genome sequencing will likely uncover an even higher prevalence of such evolution. Furthermore, our data confirm that CLL progression can occur in either a linear or a branching manner, with multiple genetic subclones evolving either in succession or in parallel (Supplementary Figure S2). These findings indicate that the linear clonal succession model of cancer evolution ${ }^{13}$ is a vast oversimplification of the changes in the genetic architecture experienced by many CLL patients.

Moreover, our data highlight the significant technologic advantages for evaluation of genomic complexity offered by the simultaneous use of whole genome and FISH analyses compared with FISH alone. Although FISH analysis is widely used in the study of CLL and it can successfully identify clinically relevant chromosomal abnormalities, ${ }^{14,15}$ FISH alone does not provide accurate insight regarding the global genomic landscape of each subclone. Patients 8 and 22, for example, had trisomy 12 as a common initial abnormality in all subclones, but subsequently underwent a marked clonal shift, characterized by multiple subclone-specific chromosomal changes. A comparable situation was found in cases where the same abnormality independently originated in different subclones (for example, patients 13 and 16 and the presence of multiple subclones with deletions of $11 \mathrm{q} 22$ and $6 \mathrm{q}$, respectively).

Ultimately we believe detailed genetic analyses such as those performed here may have relevance beyond providing biologic insights. Thus, understanding the nature of the genetic heterogeneity and the emergence of subclones are important not only in furthering knowledge of tumor biology but also potentially to improve therapy selection and clinical outcome. The discovery that, at diagnosis, genetically distinct samples and subclones already possess variably aggressive growth properties illustrates the importance of developing therapies capable of eradicating all intra-tumoral genetic subclones in order to prevent genetic evolution and recurrence. Interestingly, our genetic analysis of individual 
patients at the time of disease progression and relapse revealed that a shift in the dominant subclone can occur such that therapy may have limited efficacy if it only targets a subpopulation within the clones. Understanding the complex subclonal architecture in CLL is a critical step to developing more targeted, individualized and effective therapies for patients with CLL.

\section{Supplementary Material}

Refer to Web version on PubMed Central for supplementary material.

\section{Acknowledgments}

EB is a recipient of the Marriott Specialized Workforce Development Awards in Individualized Medicine. NEK is the recipient of the NIH awards CA95241 and CA111953. DFJ is the recipient of the NIH award CA136591.

\section{REFERENCES}

1. Anderson K, Lutz C, van Delft FW, Bateman CM, Guo Y, Colman SM, et al. Genetic variegation of clonal architecture and propagating cells in leukaemia. Nature. 2011; 469:356-361. [PubMed: 21160474]

2. Beroukhim R, Mermel CH, Porter D, Wei G, Raychaudhuri S, Donovan J, et al. The landscape of somatic copy-number alteration across human cancers. Nature. 2011; 463:899-905. [PubMed: 20164920]

3. Cottu PH, Asselah J, Lae M, Pierga JY, Dieras V, Mignot L, et al. Intratumoral heterogeneity of HER2/neu expression and its consequences for the management of advanced breast cancer. Ann Oncol. 2008; 19:595-597. [PubMed: 18272907]

4. Greenman C, Stephens P, Smith R, Dalgliesh GL, Hunter C, Bignell G, et al. Patterns of somatic mutation in human cancer genomes. Nature. 2007; 446:153-158. [PubMed: 17344846]

5. Navin N, Krasnitz A, Rodgers L, Cook K, Meth J, Kendall J, et al. Inferring tumor progression from genomic heterogeneity. Genome Res. 2010; 20:68-80. [PubMed: 19903760]

6. Notta F, Mullighan CG, Wang JC, Poeppl A, Doulatov S, Phillips LA, et al. Evolution of human BCR-ABL1 lymphoblastic leukaemia-initiating cells. Nature. 2011; 469:362-367. [PubMed: 21248843]

7. Albertson DG, Pinkel D. Genomic microarrays in human genetic disease and cancer. Hum Mo Genet. 2003; 12((Spec no 2)):R145-R152.

8. Wood LD, Parsons DW, Jones S, Lin J, Sjoblom T, Leary RJ, et al. The genomic landscapes of human breast and colorectal cancers. Science. 2007; 318:1108-1113. [PubMed: 17932254]

9. Shanafelt TD, Witzig TE, Fink SR, Jenkins RB, Paternoster SF, Smoley SA, et al. Prospective evaluation of clonal evolution during long-term follow-up of patients with untreated early-stage chronic lymphocytic leukemia. J Clin Oncol. 2006; 24:4634-4641. [PubMed: 17008705]

10. Stilgenbauer S, Sander S, Bullinger L, Benner A, Leupolt E, Winkler D, et al. Clonal evolution in chronic lymphocytic leukemia: acquisition of high-risk genomic aberrations associated with unmutated VH, resistance to therapy, and short survival. Haematologica. 2007; 92:1242-1245. [PubMed: 17666364]

11. Finn WG, Kay NE, Kroft SH, Church S, Peterson LC. Secondary abnormalities of chromosome 6q in B-cell chronic lymphocytic leukemia: a sequential study of karyotypic instability in 51 patients. Am J Hematol. 1998; 59:223-229. [PubMed: 9798660]

12. Kay NE, Geyer SM, Call TG, Shanafelt TD, Zent CS, Jelinek DF, et al. Combination chemoimmunotherapy with pentostatin, cyclophosphamide, and rituximab shows significant clinical activity with low accompanying toxicity in previously untreated B chronic lymphocytic leukemia. Blood. 2007; 109:405-411. [PubMed: 17008537]

13. Nowell PC. The clonal evolution of tumor cell populations. Science. 1976; 194:23-28. [PubMed: 959840] 
14. Dohner H, Stilgenbauer S, Benner A, Leupolt E, Krober A, Bullinger L, et al. Genomic aberrations and survival in chronic lymphocytic leukemia. N Engl J Med. 2000; 343:1910-1916. [PubMed: 11136261]

15. Dewald GW, Brockman SR, Paternoster SF, Bone ND, O'Fallon JR, Allmer C, et al. Chromosome anomalies detected by interphase fluorescence in situ hybridization: correlation with significant biological features of B-cell chronic lymphocytic leukaemia. Br J Haematol. 2003; 121:287-295. [PubMed: 12694251] 

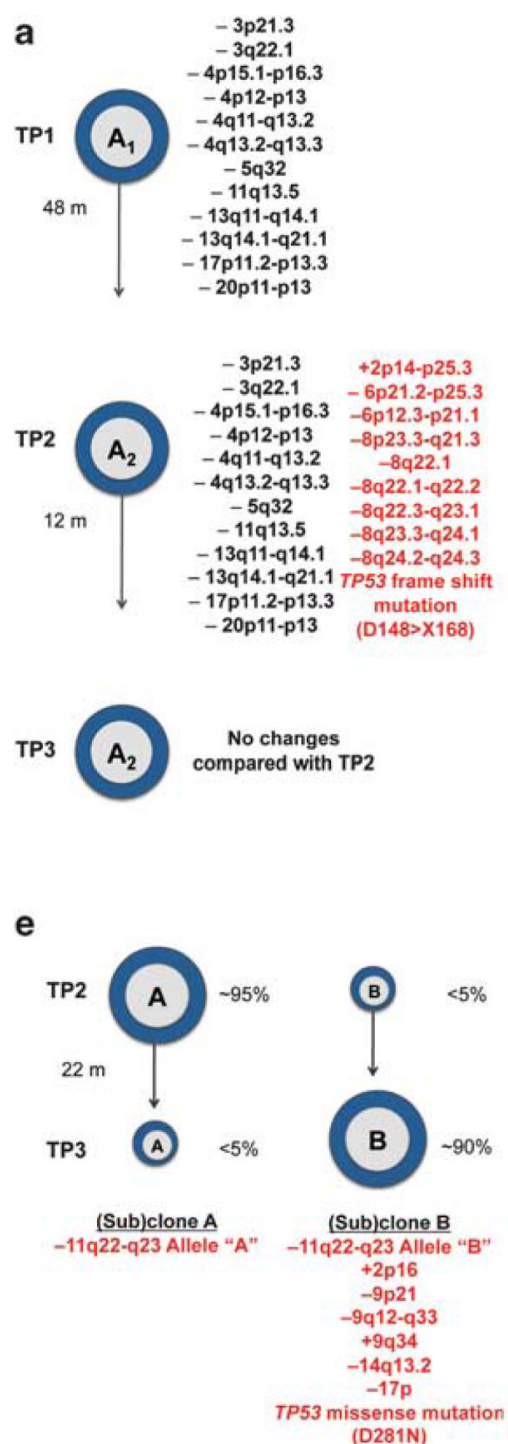

\section{Figure 1.}

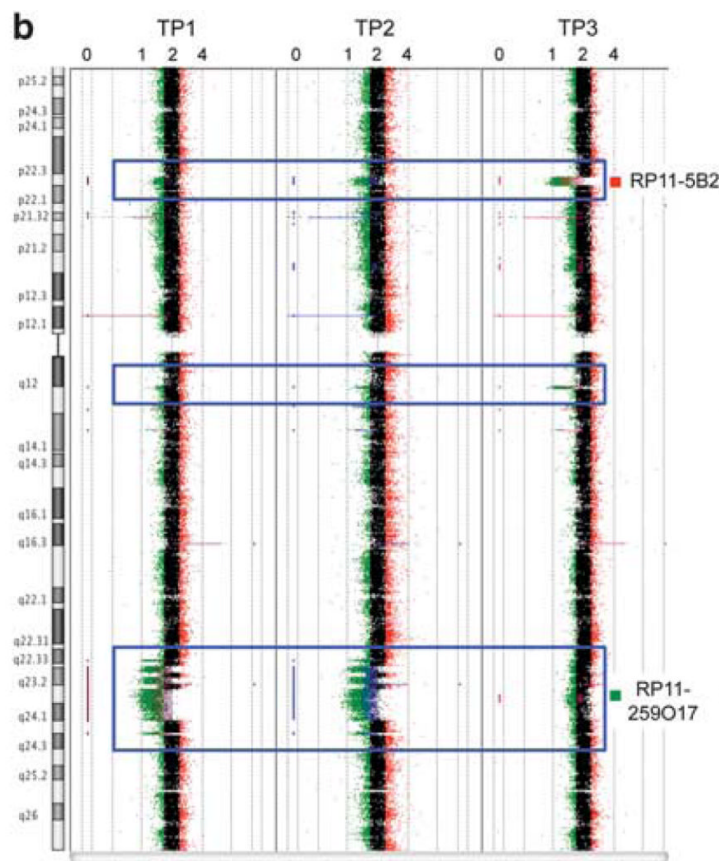

f

TP2

TP3

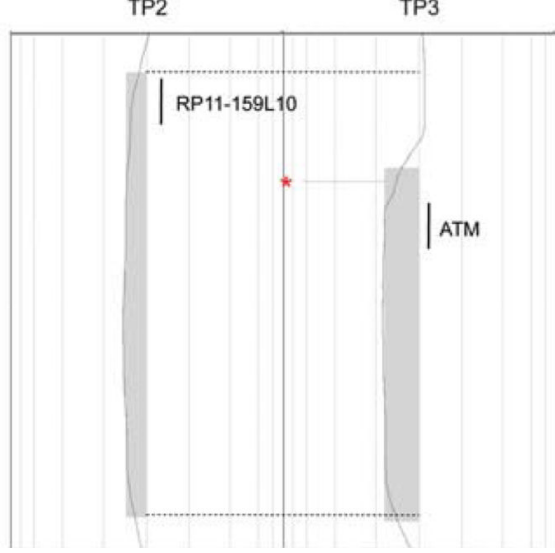

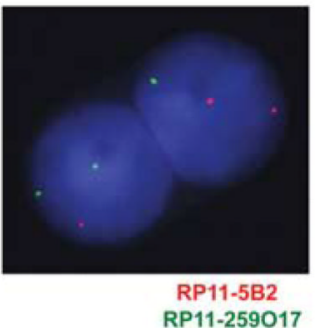

d Original clone

$+12$
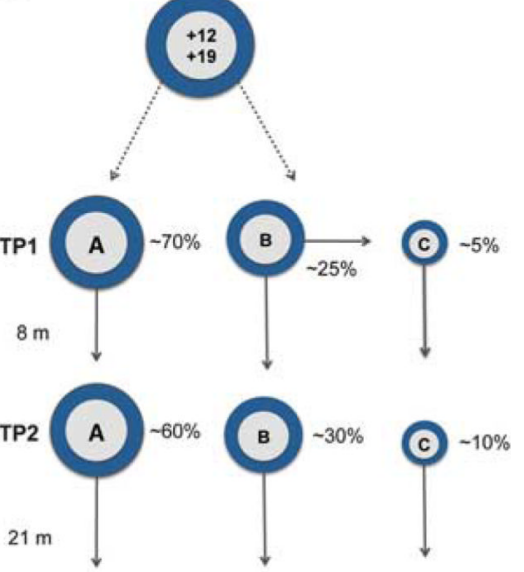

TP3
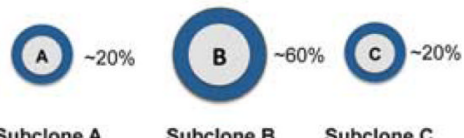

\begin{tabular}{|c|c|}
\hline Subclone B & Subclone C \\
\hline +12 & +12 \\
\hline+19 & +19 \\
\hline$-5 q 14.3$ & $-5 q 14.3$ \\
\hline$-5 q 33.2$ & $-5 q 33.2$ \\
\hline$-6 p 22.2$ & $-6 p 22.2$ \\
\hline$-6 q 12$ & $-6 q 12$ \\
\hline$-8 \mathrm{p} 12$ & $-8 \mathbf{p} 12$ \\
\hline$-13 q 14.3$ & $-13 q 14.3 \times 2$ \\
\hline
\end{tabular}

CLL progression can occur either in a linear or in a branching manner, with multiple genetic subclones evolving either in succession or in parallel. (a) Linear evolution with the maintenance of the initial CNA and the subsequent acquisition of additional CNAs in the same subclone found on patient 17. The emerging CNAs not found in previous TPs are shown in red. (b-f) Branching clonal progression, with multiple genetic subclones evolving in parallel. (b) aCGH plots show the evolution of chromosome 6 through TPs (TP1 - P3) on patient 8 . Deletions of 6p22.2 and 6q12 -q13 become more evident in the later TP3, whereas multiple deletions on 6q23-q24 decrease in prevalence over time (blue boxes). (c) Custom FISH probes were designed in these regions and used to confirm the simultaneous presence of multiple subclones. (d) Three subclones were found on patient 8 based on aCGH and FISH analyses. All subclones shared CNAs on chromosomes 12 and 19. In red are shown the abnormalities subclone-specific. There is a marked clonal shift between TP1 and TP2 and between TP2 and TP3. Subclone 'A' is the dominant clone in TP1 and TP2, but its prevalence is significantly reduced at TP3. Conversely, subclone 'B' emerged at TP3 becoming the dominant clone. The genetic architecture and prevalence of each clone was calculated using multiplex FISH assays. (e) Two (sub) clones with no apparent common 
CNAs were found on patient 16 . Clone 'A' was characterized by deletion of 11q22-q23 and was the dominant clone at TP2 but disappeared or was below the detection FISH detection threshold at TP3. Conversely, clone 'B' was undetectable at TP2 by aCGH or FISH, but became the dominant clone at TP3. (f) Although both clones in patient 8 harbored deletion of 11q22-q23, they were independent clones, as the affected regions (showed by gray blocks at the bottom panel) did not share any of the breakpoints in aCGH analysis (breakpoint positions found in TP2 are indicated by broken lines and compared with TP3; showed at the right panel). Data were confirmed using a validation FISH assay analyzing the differentially affected region between clones (BAC probe RP11-159L10). In addition, a biallelic deleted CNV (indicated with a red *) in TP3 but not in TP2 indicates that different alleles of 11q22q23 were affected in the two clones. The physical positions of probe RP11-159L10 and ATM gene are shown. In all cases analyzed, the emerging CNAs not found in previous TPs are shown in red. The genetic architecture and prevalence of each genetic clone was calculated using multiplex FISH assays. TP1-TP3: TPs 1-3. The time interval in months (m) between samples is shown. 
a
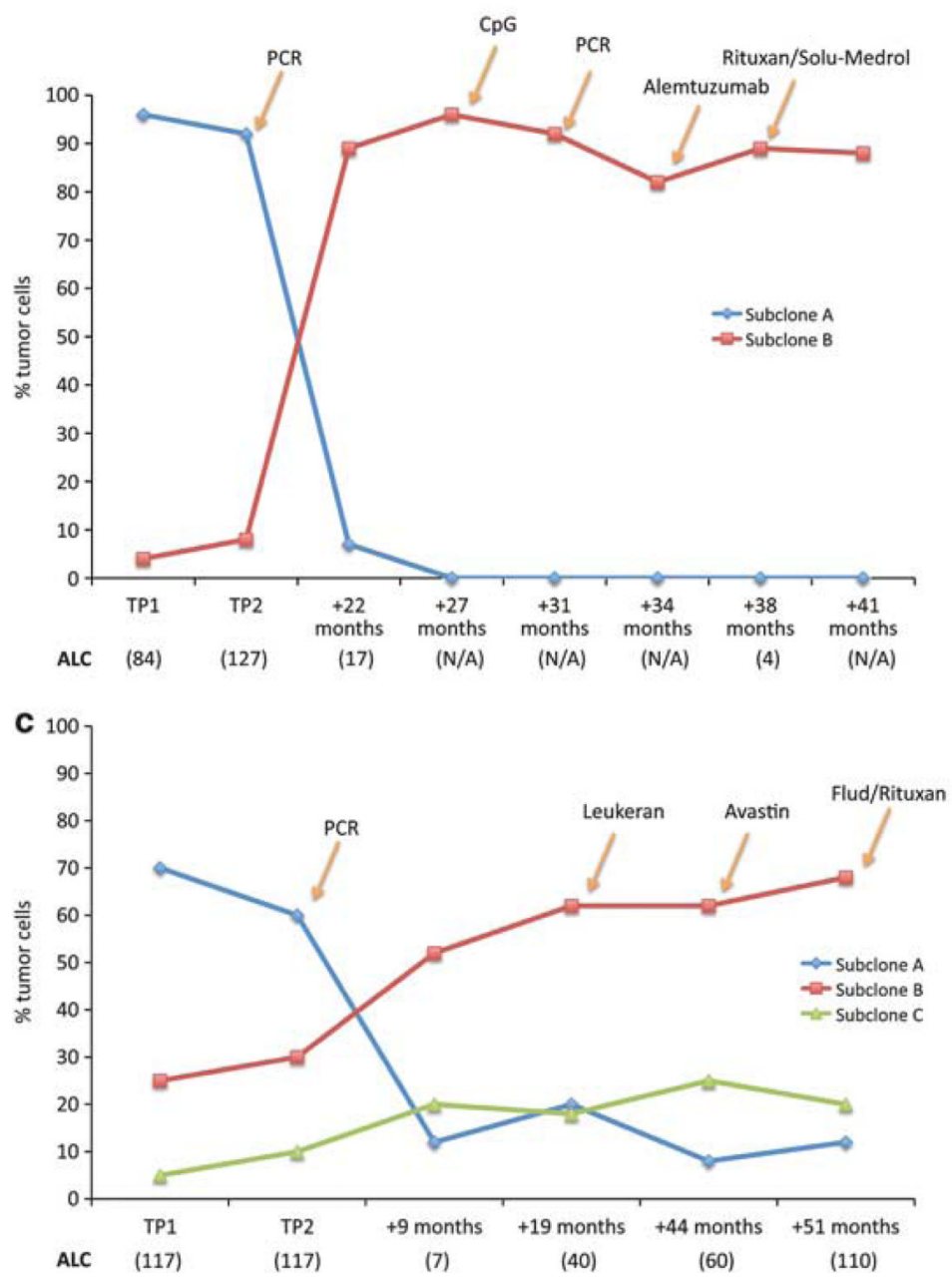

b
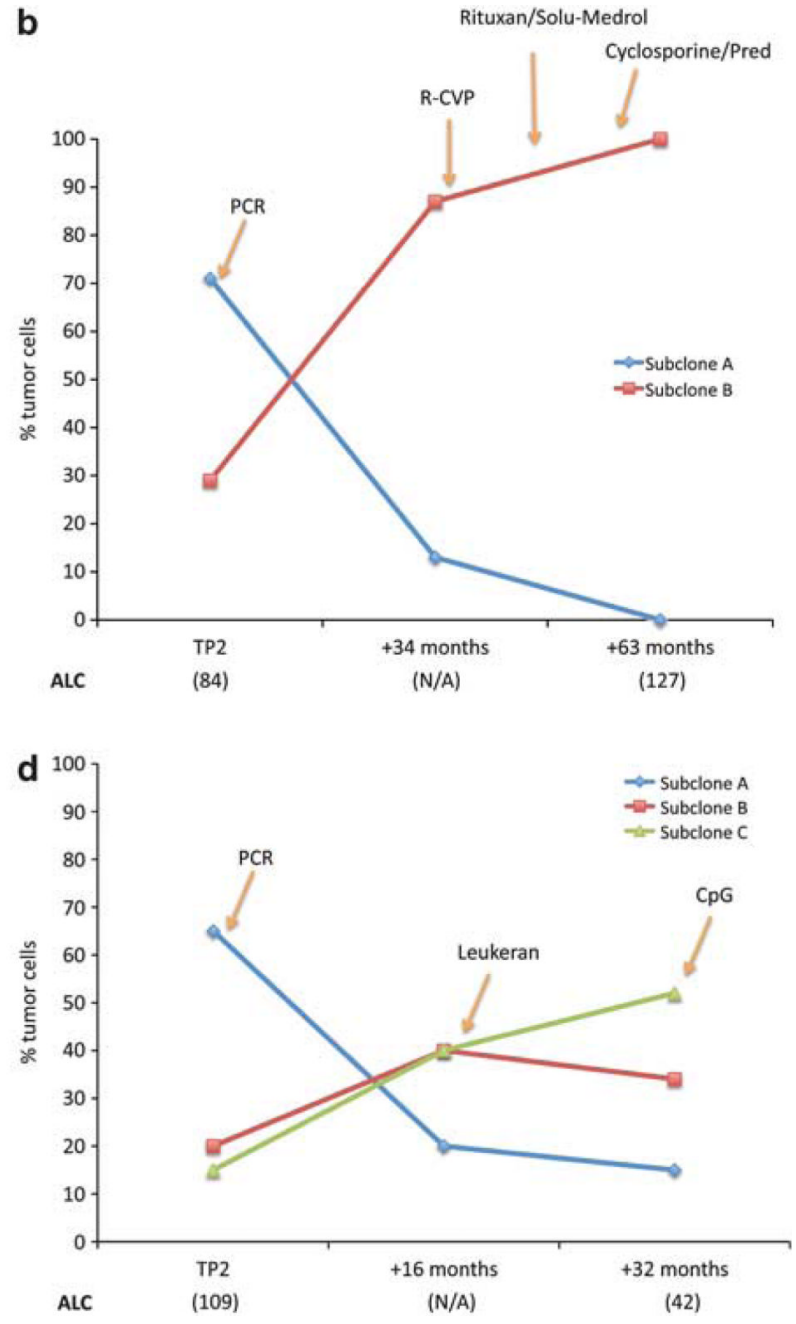

Figure 2.

Minor clones emerge preferentially after relapse of therapy. Pattern of clonal evolution in four CLL cases. The clonal pattern established at diagnosis was then studied over time in these cases. Clonal evolution was noted after initial therapy with PCR as well as after secondary therapies. In all four cases the emerging subclone shows resistance to multiple treatments. (a) Patient 16, (b) patient 22, (c) patient 8 and (d) patient 13. The charts show the prevalence of each subclone in the tumor as calculated by FISH. Different TPs were analyzed and here are indicated as TP1, TP2 and follow-up therapies (time in months after initial therapy are indicated). Yellow arrows indicate when additional therapies were used. Where available, the absolute lymphocyte counts (ALC) are shown in parentheses below each TP on the $x$-axis. N/A, not available. 